\title{
AÇÃO HIPOGLICEMIANTE DO EXTRATO AQUOSO DO CAULE DA BAUHINIA FORFICATA (MORORÓ) EM MODELOS EXPERIMENTAIS DIABÉTICOS
}

\author{
HYPOGLYCAEMIC ACTION OF THE AQUEOUS EXTRACT OF THE \\ STEM OF BAUHINIA FORFICATA IN EXPERIMENTAL MODELS \\ INDUCED BY ALOXANA.
}

\author{
Patrícia Pereira da Silva Dias ${ }^{1}$ \\ Francisco Eduardo Ferreira Alves ${ }^{2}$ \\ Jose Diego Oliveira Alves ${ }^{3}$ \\ Kellyanne Pereira da Silva ${ }^{4}$ \\ Maria Iranilda Silva Magalhães ${ }^{5}$
}

RESUMO: A diabetes mellitus é causada por uma disfunção nas ilhotas pancreáticas que irá desencadear o impedimento da glicose nas células. As plantas medicinais, desde a antiguidade até os dias atuais, são utilizadas como métodos de tratamento de diversas patologias, promovendo respostas fisiológicas nos organismos vivos. O uso destas práticas perpetuou-se, sendo utilizada recorrentemente em todo o mundo, os conhecimentos que são passados por gerações são de grande relevância para a ciência. O presente trabalho buscou investigar a ação hipoglicemiante do extrato aquoso do caule da Bauhinia forficata em ratos, da linhagem Wistar, diabéticos induzidos por Aloxana monohidratada. Para isso, utilizou-se de ratos divididos em 4 distintos grupos com a utilização diária da Bauhinia: no grupo 1 foram administradas doses de $200 \mathrm{mg}$ do extrato aquoso da Bauhinia forficata em ratos diabéticos; no grupo 2 administrou-se $500 \mathrm{mg}$ de metformina (fármaco hipoglicemiante) nos ratos diabéticos, levando-os em alguns dias de glicemia superior a $500 \mathrm{mg} / \mathrm{dL}$ trocou-se por $0,2 \mathrm{~mL}$ de insulina para controle da quantidade de óbitos; no grupo 3 foram administrados $100 \mathrm{mg}$ do extrato em ratos diabéticos e no grupo 4 não administrou-se fármaco hipoglicemiante, nem tampouco extrato, utilizando-se de ratos saudáveis para controle. As análises foram feitas em dias úteis nos 4 grupos, cada um com 5 ratos, e coletou-se os dados por 17 dias. Obteve-se o número de 5 ratos durante as induções. A pesquisa foi

\footnotetext{
${ }^{1}$ Graduação em Biomedicina pela Faculdade Santa Maria.

${ }^{2}$ Graduação em Biomedicina pela Faculdade Santa Maria.

${ }^{3}$ Graduação em Biomedicina pela Faculdade Santa Maria.

${ }^{4}$ Graduação em Biomedicina pela Faculdade Santa Maria.

${ }^{5}$ Biomédica Docente da Faculdade Santa Maria.
} 
satisfatória, observada através do grupo 1, que obteve a maior dosagem do extrato, $200 \mathrm{mg} / \mathrm{kg}$, mostrando uma diminuição de glicemia de $38 \mathrm{mg} / \mathrm{dL}$ do grupo. A Bauhinia forficata pôde desempenhar função hipoglicemiante e, através do objetivo desta pesquisa, buscou-se apresentar resultados que demonstrassem a importância da continuidade da busca por dosagens específicas, estudando seus efeitos colaterais e tóxicos, que trarão importantes benefícios para o ramo científico.

Palavras chave: Plantas medicinais. Bauhinia forficata. Diabetes mellitus.

ABSTRACT: The diabetes mellitus is caused by a dysfunction in the pancreatic islets that will trigger the impairment of glucose in the cells. Medicinal plants, from antiquity to the present day, are used as methods of treatment of various pathologies, promoting physiological responses in living organisms. The use of these practices has been perpetuated, being used recurrently throughout the world. The knowledge that is passed by generations is of great relevance to science, because it is aggregated relevant information about biological phenomena. The empirical knowledge is of total interest to science, because it is the knowledge of people, being mostly lay on the scientific branch, however, it has knowledge as some scientists. The present work sought to investigate the hypoglycemic action of the aqueous extract of the stem of Bauhinia forficata in rats, of the Wistar lineage, diabetics induced by Alloxana monohydrate. Daily doses of $200 \mathrm{mg}$ of the aqueous extract of Bauhinia forficata in diabetic rats in Group 1, in Group 2, administered $500 \mathrm{mg}$ of metformin (hypoglycemic drug) in diabetic rats, which in some days of glycemia higher than $500 \mathrm{mg} / \mathrm{DL} 0.2 \mathrm{ML}$ of insulin was exchanged in the group, $100 \mathrm{mg}$ of the extract was administered in diabetic rats and in Group 4 it was not administered a hypoglycaemic drug or extract, being healthy rats for control. The analyses were done on weekdays of the 4 groups, each with 5 rats, induced diabetes mellitus through the Alloxan and collected the data for 17 days. The literature mentions that varying indices of mortality after diabetic induction are considerable, because there are multiple factors that are able to change the effects of the drug and the sensitivity of the host, such as: fasting time, animal weight, hydration status, infusion speed, diet, route of administration. The number of 5 rats was obtained during the inductions. The study was satisfactory, observed through group 1, which obtained the highest dosage of the extract, $200 \mathrm{mg} / \mathrm{kg}$, showing a decrease in glycemia of 38 $m g / D L$ of the group. This line of research should be continued, since science needs a thorough and long-term study about this extract, to determine specific dosages in which they can be used without causing toxicity, and thus contribute to the society For the control or even the cure of this aggressive pathology. The Bauhinia Forficata was able to perform the hypoglycemic role, through this research, it continues to research specific dosages, studying its side and toxic effects, which will bring important benefits to the scientific branch.

Key words: Medicinal plants. Bauhinia Forficata. Diabetes mellitus. 


\section{INTRODUÇÃO}

As plantas medicinais são utilizadas como métodos de tratamento de diversas patologias, promovendo respostas fisiológicas nos organismos vivos. Conhecimentos empíricos sobre estes métodos são passados de geração em geração, sem o estudo de doses e efeitos colaterais. Estudos etnobotânicos apontam a grande relevância que tem a utilização de plantas, que por meio dos seus princípios ativos causam determinados eventos biológicos por diversos mecanismos já comprovados em literatura (JARADAT et al., 2016; GOIS et al., 2016).

Essas plantas são vegetais que podem ser utilizados para fins terapêuticos, possuindo constituintes químicos que apresentam uma ação eficaz para determinada patologia. No Brasil, a prática curandeira através de vegetais é favorecida pela grande diversidade de espécies e diferentes biomas que o país oferece, que podem ser utilizadas para uma prática preventiva ou no tratamento de patologias. Também podem ser correlacionados pelo alto custo de medicamentos, sendo a utilização de plantas como remediação com custeio baixo e facilidade de acesso (PEREIRA et al., 2015).

Conhecida como 'Pata de Vaca' ou mororó, a Bauhinia forficata é uma angiosperma, pertencente à família Fabaceae, distribuída em regiões com clima úmido. Nativa da Argentina, muito encontrada no Brasil, usadas popularmente em preparações terapêuticas na medicina tradicional utilizando seu caule e folhas, por suas propriedades hipoglicêmicas e diuréticas que estão em recentes análises para se comprovar se há ação fisiológica para diminuição da glicemia (BUSZNIEZ, 2017).

As suas folhas são utilizadas na forma de chá, mais comumente na comunidade rural. Este tipo de utilização vem sido relatado em estudos feitos por Trojan-Rodrigues et al. (2012), onde apresentam-se análises etnobotânicas que comprovaram como a Bauhinia forficata apresentou superioridade no tratamento da diabetes mellitus no Estado do Rio Grande do Sul. Além disso, a Bauhinia forficata 
está entre as plantas medicinais mais vendidas em todo o Brasil (MARQUES et al., 2013).

A Diabetes mellitus é causada por uma disfunção nas ilhotas pancreáticas que irá desencadear o impedimento da glicose nas células. Tal desregulação não é bem compreendida a nível celular, mas sim pelo estudo da alta taxa de glicose circulante no sangue periférico. Patologia progressiva, precisamente caracterizada pela hiperglicemia persistente, tendo como consequência disfunções metabólicas nos carboidratos, proteínas e gorduras, fazendo com que haja uma drástica diminuição na ação ou secreção da insulina (GE LI et al., 2016).

O diabetes mellitus tipo 2 é um grupo heterogêneo de distúrbios metabólicos que se dá por defeitos no receptor de insulina, mas esta, ainda continuará sendo produzida nas células $\beta$, permanecendo inalteradas. Os tratamentos disponíveis estão agregados com a diminuição a resistência da insulina, e o melhoramento da função das células $\beta$ pancreáticas se dá através da dieta, hipoglicemiantes orais, exercícios físicos, anti-hiperglicemiantes com/ou agrupados a drogas antiobesidade (COSTA et al., 2017).

O presente trabalho buscou investigar a ação hipoglicemiante do extrato aquoso do caule da Bauhinia forficata (Mororó) em ratos, da linhagem Wistar, diabéticos induzidos por Aloxana monohidratada. Devido à falta de conhecimentos acerca da Bauhinia forficata e sua ação hipoglicemiante, o presente trabalho justifica-se com a finalidade de esclarecer se há ou não o controle glicêmico no diabético com a utilização do extrato, utilizando ratos diabéticos e garantindo que outros fatores como dieta e exercícios não interferissem na análise.

\section{MATERIAL E MÉTODOS}

Trata-se de um estudo do tipo experimental que visa testar a ação hipoglicemiante do extrato aquoso do caule da Bauhinia forficata em ratos, da linhagem Wistar, diabéticos induzidos por Aloxana. 
A Bauhinia forficata foi coletada em agosto de 2018 de uma plantação da zona rural da cidade de Sousa, no estado da Paraíba, Brasil. Coletou-se apenas a parte de interesse para a pesquisa, o caule. Temperatura solar de 30-35 graus, em seguida preparado a secagem do material vegetal, maceração e obtenção do extrato etanoico para filtração e a concentração em rotoevaporador e banho maria, o extrato bruto foi colocado em solução aquosa. Obteve-se $765 \mathrm{~g}$ do extrato bruto concentrado, acrescentando-se $1000 \mathrm{~mL}$ de água filtrada.

Os experimentos foram realizados em ratos modelo Wistar de ambos os sexos, com peso médio entre $200 \mathrm{~g}-300 \mathrm{~g}$ com mais de 60 dias de nascimento, considerando-se ratos jovens, obtidos do biotério da Faculdade Santa Maria de acordo com as normas do Comitê de Ética em Utilização Animal da Instituição durante 17 dias, com documento em anexo de número 001/2018 em anexo C.

Os animais foram mantidos no biotério central da instituição, em gaiolas de polietileno, por um período de 3-7 dias em análise de procedimento. Temperaturas constantes de $20-25^{\circ} \mathrm{C}$, controladas por equipamentos de ar condicionado e limpeza em suas gaiolas periodicamente, ciclo claro/escuro de 12 horas, sendo a fase clara de 06 às 18 horas, recebendo ração padrão Nuvilab® e água ad libitum disponíveis em garrafas de polietileno com bicos de inox.

O extrato bruto ficou em maceração por 72 horas no etanol à $70 \%$. Os ensaios foram realizados com doses de $100 \mathrm{mg}$ de extrato para cada $\mathrm{kg}$ de animal e $200 \mathrm{mg}$ de extrato para cada $\mathrm{kg}$ de animal. O solvente utilizado foi a água filtrada.

Foram divididos aleatoriamente em cinco grupos. Separados: G1 animais com diabetes induzidos com Aloxana com dose de $200 \mathrm{mg}$ do extrato da Bauhinia forficata (número de 5 animais); G2 animais com diabetes induzidos com Aloxana utilizando a droga antidiabética Cloridrato de Metformina $(500 \mathrm{mg} / \mathrm{kg}$ ) (número de 5 animais); G3 animais com diabetes induzidos com Aloxana com dose de $100 \mathrm{mg}$ do extrato da Bauhinia forficata (número de 5 animais); G4 animais controle sem diabetes e sem utilização da Aloxana (número de 5 animais); G5 animais com diabetes induzidos com Aloxana com dose de $50 \mathrm{mg}$ do extrato da Bauhinia forficata (número de 5 animais).

Após a segunda indução, 5 ratos foram a óbito, havendo uma redistribuição dos grupos, tendo 4 grupos: G1 animais com diabetes induzidos com Aloxana com 
dose de $200 \mathrm{mg}$ do extrato da Bauhinia forficata (número de 5 animais); G2 animais com diabetes induzidos com Aloxana utilizando a droga antidiabética Cloridrato de Metformina (500 mg/kg) (número de 5 animais); G3 animais com diabetes induzidos com Aloxana com dose de $100 \mathrm{mg}$ do extrato da Bauhinia forficata (número de 5 animais) e G4 animais controle sem diabetes e sem utilização de fármacos (número de 5 animais).

A escolha desses animais foi baseada em fácil manuseio, ocupação de pequenos espaços para se trabalhar simultaneamente com vários grupos experimentais, alta resistência a infecções, redução de custos, e por apresentarem grandes semelhanças clínicas, laboratoriais e histopatológicas com o diabetes humano.

A Aloxana é uma pirimidina, sua estrutura se assemelha ao ácido úrico e a glicose. Suas propriedades diabetogênicas possuem ação seletiva e destrutiva sobre as células $\beta$ pancreáticas. A indução ao diabetes mellitus tipo 2 em animais experimentais, está diretamente relacionada à formação de radicais superóxido e hidroxila que são tóxicos para tais células, que se leva à degeneração e morte definitiva das mesmas (LIMA et al., 2001). Para indução química do diabetes tipo 2 , os animais foram mantidos em jejum sólido por 24 horas. Após este período foi realizado a pesagem e dosagem da glicemia de cada indivíduo na pré-indução. Os níveis de glicose foram monitorados por meio de glicosímetro comercial OneTouch Ultra (Johnson \& Johnson®).

A solução de Aloxana monoidratada (Sigma-Aldrich Inc, St Louis, MO, USA) foi comprada através da empresa Interprise Comércio e Serviços Tecnológicos LTDA, que trouxe o fármaco advinda internacionalmente, conservando-se a temperatura de $-20^{\circ} \mathrm{C}$ e em recipiente adequado até a preparação da solução. Foi preparada minutos antes da aplicação por meio da diluição do fármaco em solução salina $0,9 \%$, com uma concentração de $40 \mathrm{mg} / \mathrm{mL}$. O preparo desta solução foi realizado em ambiente escuro, utilizando um frasco âmbar envolvido por papel alumínio para evitar contato da luz, visto que o composto é fotossensível. Além disso, após a diluição, o frasco foi mantido refrigerado até o momento da aplicação. Os animais receberam dose única de $120 \mathrm{mg} / \mathrm{kg}$ de Aloxana via intraperitoneal. Após uma hora e meia, a alimentação foi reintroduzida aos animais. Aqueles que atingiram 
glicemia superior a $200 \mathrm{mg} / \mathrm{dL}$ foram considerados diabéticos. Os animais que não obtiveram este índice foram novamente submetidos ao protocolo de indução.

Foram analisadas e administradas todas as dosagens em todos os dias úteis, totalizando 17 dias. Nos 4 grupos, fez-se a análise da glicemia e a massa corporal a cada dois dias úteis, utilizando o glicosímetro, através de uma gota de sangue coletada do rabo dos ratos e sua massa corporal através de uma balança de precisão de gramas. Sua alimentação era de $550 \mathrm{~g}$ de ração e $800 \mathrm{~mL}$ de água em cada grupo, fazendo-se as pesagens diárias e reabastecendo para que a quantidade diária seja a mesma inicialmente. A utilização da Bauhinia forficata foi feita diariamente, assim como no grupo que foi utilizado a Metformina (droga utilizada para controle glicêmico). Houve alteração da utilização da Metformina para ratos que apresentassem glicemia maior que $500 \mathrm{mg} / \mathrm{dL}$, administrando $20 \mu \mathrm{L}$ de insulina, para que não houvesse maior quantidade de óbitos.

A pesquisa foi desenvolvida na Faculdade Santa Maria (FSM). Os experimentos foram feitos no Laboratório de Habilidades Cirúrgicas de Análises Clínicas da instituição, que fica localizado na cidade de Cajazeiras-PB.

Os critérios utilizados foram ratos Wistar de ambos os sexos que apresentaram peso entre $200 \mathrm{~g}$ - $300 \mathrm{~g}$ em número de 25 ratos, divididos em 4 grupos. Foram excluídos da pesquisa animais que ao utilizar a Aloxana não ficaram diabéticos. A utilização do extrato foi feita após duas induções do diabetes mellitus, contabilizando 15 dias de observação e análise glicêmica, para assim começar o experimento.

Antes de cada procedimento as bancadas e os aparelhos utilizados foram higienizados com álcool à $70 \%$. A pesquisa seguiu em conformidade e foi conduzida em obediência às normas e diretrizes bioéticas vigentes para ensaios envolvendo seres vivos: animais (Guide for the Care and Use of Laboratory Animals, do NIH National Institute of Health-EUA, 1996; Lei Federal № 11.794/2008; Conselho Nacional de Controle de Experimentação - CONCEA); e integridade da fauna e flora (Lei Federal No 9605/1998) (MACHADO et al., 2004). O projeto foi aprovado pelo Comitê de Ética em Pesquisa Animal da Faculdade Santa Maria - FSM, com o número 001/2018. 
Os dados e informações foram analisados no SPSS (Statistical Package for the Social Sciences) versão 23, utilizando estatísticas descritivas de média, mediana, desvio padrão de + ou- 2, valores mínimos e máximos, além de frequência e porcentagem.

\section{RESULTADOS E DISCUSSÕES}

Houve duas induções da Alloxana, por alguns ratos não ter atingido a hiperglicemia, deste modo, houve uma baixa de peso brusca, diarreia e em alguns casos cegueira, justificada pelo aumento progressivo nos níveis de glicose dos ratos durante o experimento caracterizando quadro clínico diabético permanente e grave provocado pela Aloxana. A variância de peso era de $200 \mathrm{~g}$ a $300 \mathrm{~g}$. Na segunda indução, contabilizou-se 5 óbitos por hiperglicemia, continuando a pesquisa com 20 ratos, redistribuindo-os em quatro grupos. A metformina não se mostrou eficiente na dosagem de $500 \mathrm{mg} / \mathrm{kg}$, substituída em alguns dias de glicemia maior que 500 $\mathrm{mg} / \mathrm{dL}$ por $0,2 \mathrm{~mL}$ de insulina. 
Figura 1: Análise glicêmica de ratos modelo Wistar.

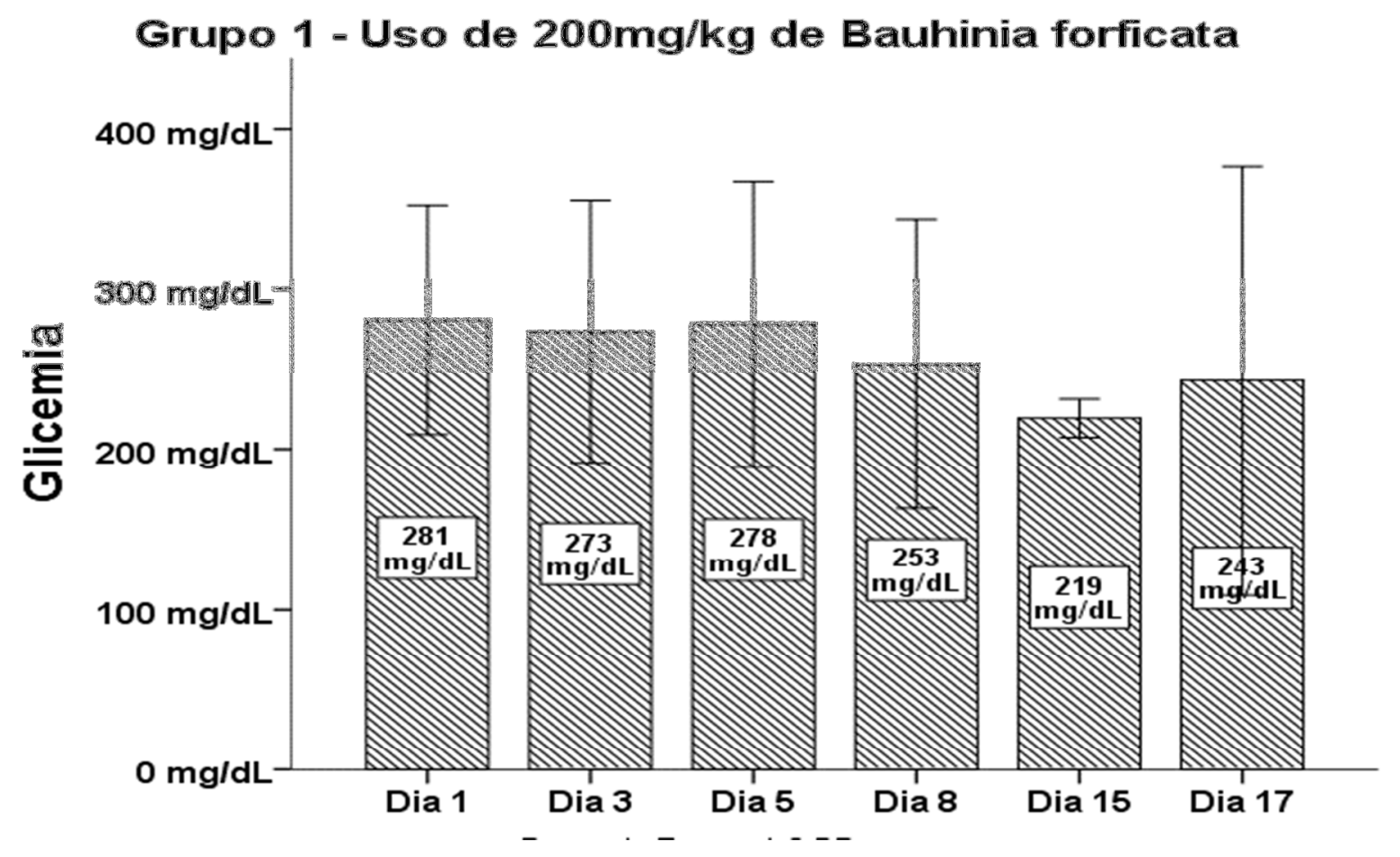

Figura 1A: Análise glicêmica de ratos diabéticos que fazem o uso de $200 \mathrm{mg}$ de Bauhinia forficata. 


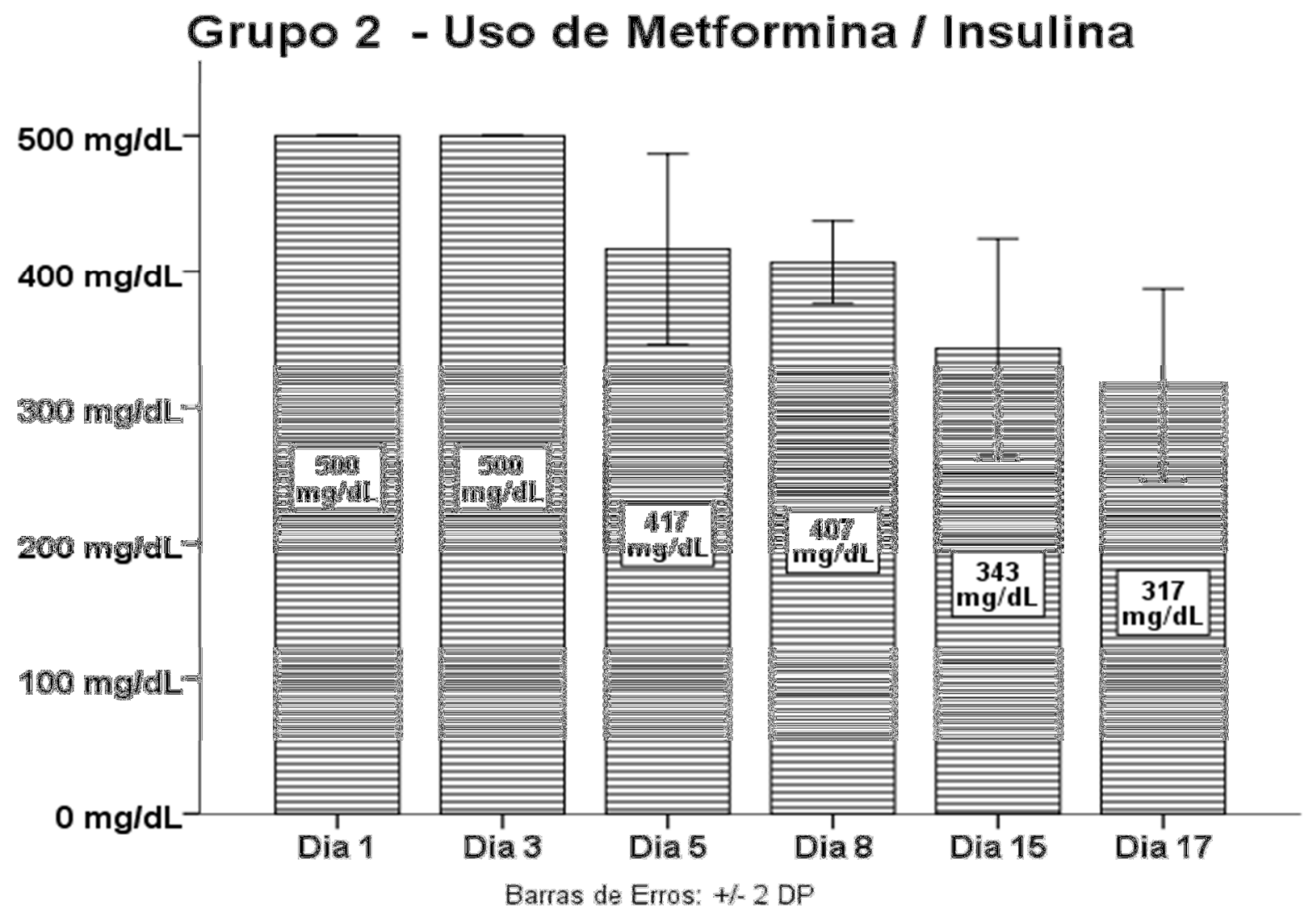

Figura 1B: Análise Glicêmica de ratos diabéticos que fazem o uso de $500 \mathrm{mg}$ de metformina/ $0,2 \mathrm{~mL}$ insulina. 


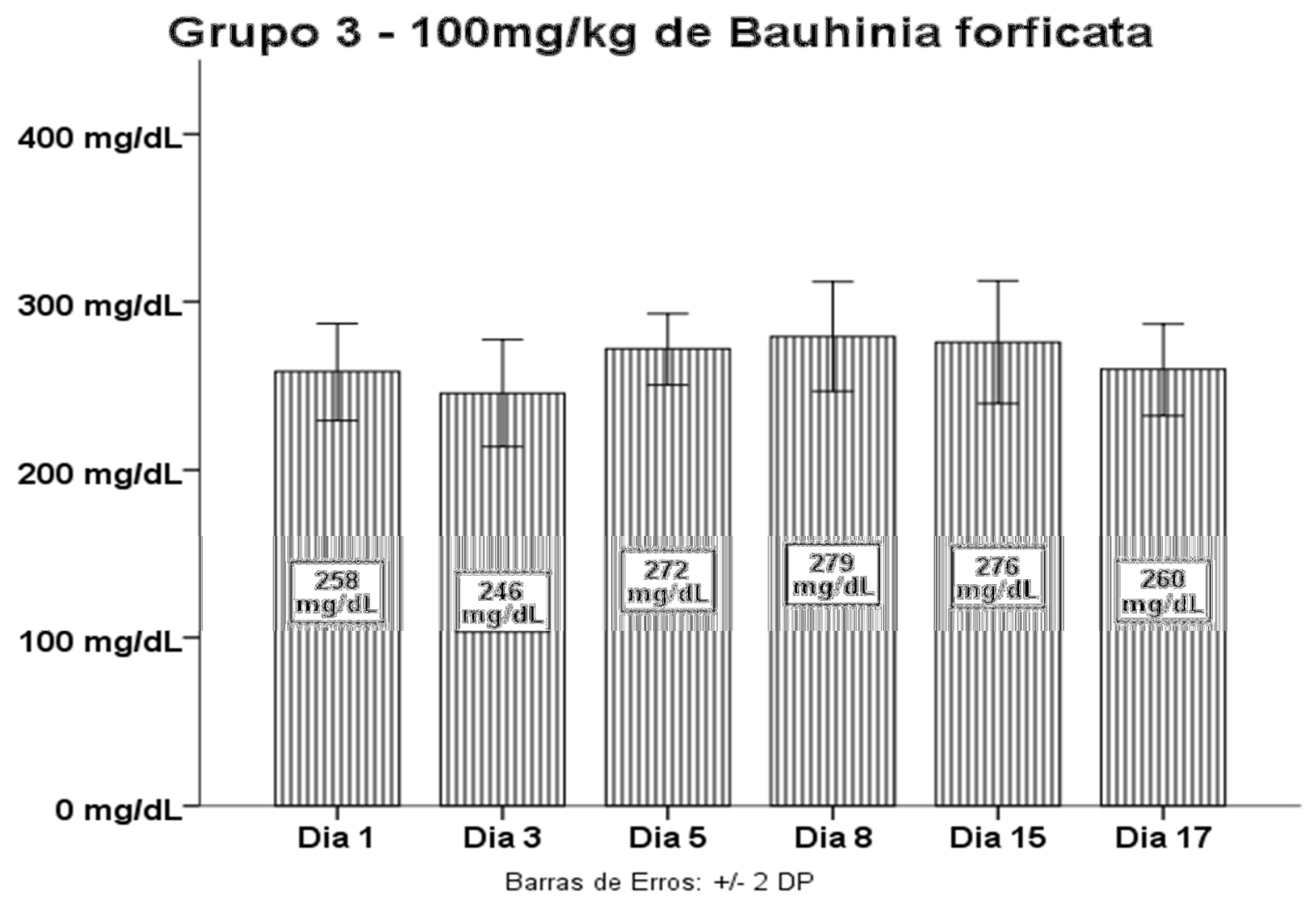

Figura 1C: Análise glicêmica de ratos diabéticos que fazem o uso de $100 \mathrm{mg}$ de Bauhinia forficata. 


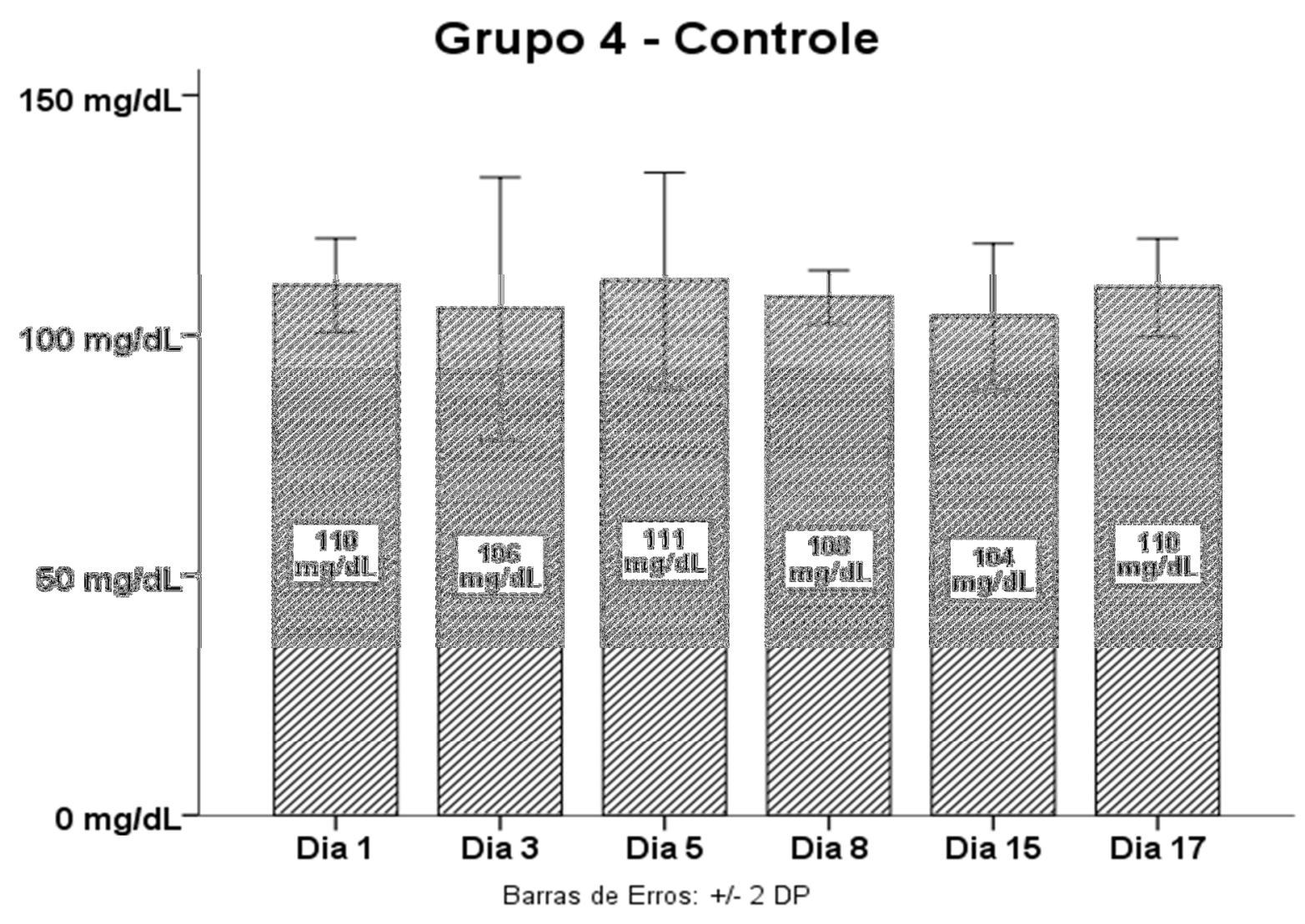

Figura 1D: Análise Glicêmica de ratos não diabéticos para controle.

$\mathrm{Na}$ figura $1 \mathrm{~A}$, ilustrando o grupo 1 da pesquisa, foi administrado $200 \mathrm{mg} / \mathrm{kg}$ do extrato aquoso da Bauhinia forficata, de modo que se relacionou com a glicemia em $\mathrm{mg} / \mathrm{dL}$ dos ratos em dias alternados. Obteve-se diante análise, resultados satisfatórios, uma vez que a variância na glicemia foi significativa para a pesquisa, pois a média da glicemia do grupo iniciou-se com $281 \mathrm{mg} / \mathrm{dL}$ e concluiu-se com 243 $\mathrm{mg} / \mathrm{dL}$, de modo que houve uma diminuição de $38 \mathrm{mg} / \mathrm{dL}$ de média da glicemia do grupo durante o período de 17 dias de análise.

$\mathrm{Na}$ figura 1B, na qual ilustra o grupo 2, administrou-se $500 \mathrm{mg} / \mathrm{kg}$ do fármaco Cloridrato de metformina/insulina, utilizado habitualmente por pessoas diabéticas como hipoglicemiante, sendo relacionado a glicemia em $\mathrm{mg} / \mathrm{dL}$ em diferentes dias no decorrer da pesquisa. Os resultados encontrados satisfizeram com o que se era esperado, tendo uma baixa significativa da glicemia, com uma glicemia inicial de 500 $\mathrm{mg} / \mathrm{dL}$ e ao seu término $317 \mathrm{mg} / \mathrm{dL}$, obtendo-se uma diminuição gradual de 183 
$\mathrm{mg} / \mathrm{dL}$ de média glicêmica do grupo, mostrando-nos que o aumento de suas dosagens, levaram a um controle glicêmico não só pela utilização da metformina, mas também pelo uso da insulina.

$\mathrm{Na}$ figura 1C, ilustra-se o grupo 3, administrado $100 \mathrm{mg} / \mathrm{kg}$ do extrato da Bauhinia forficata, relacionando-se a glicemia em $\mathrm{mg} / \mathrm{dL}$ em dias alternados, tendo como resultados uma variação muito menor em comparação ao grupo 1, da figura $1 \mathrm{~A}$, pois a glicemia do grupo iniciou-se com $258 \mathrm{mg} / \mathrm{dL}$ e encerrou-se com 260 $\mathrm{mg} / \mathrm{dL}$, tendo uma diminuição um aumento de sua média de $2 \mathrm{mg} / \mathrm{dL}$ do dia 1 ao dia 17.

Na figura 1D, ilustra-se grupo 4, também chamado de grupo controle, onde não foi administrado nenhum fármaco e nem induzido a diabetes pela Aloxana monohidratada, sendo o grupo para mostrar-nos a glicemia de ratos saudáveis, tendo uma glicemia inicial do grupo de $110 \mathrm{mg} / \mathrm{dL}$ e encerrando-se com a mesma dosagem de glicemia.

Figura 2. Avaliação da massa corporal de ratos diabéticos por Aloxana.

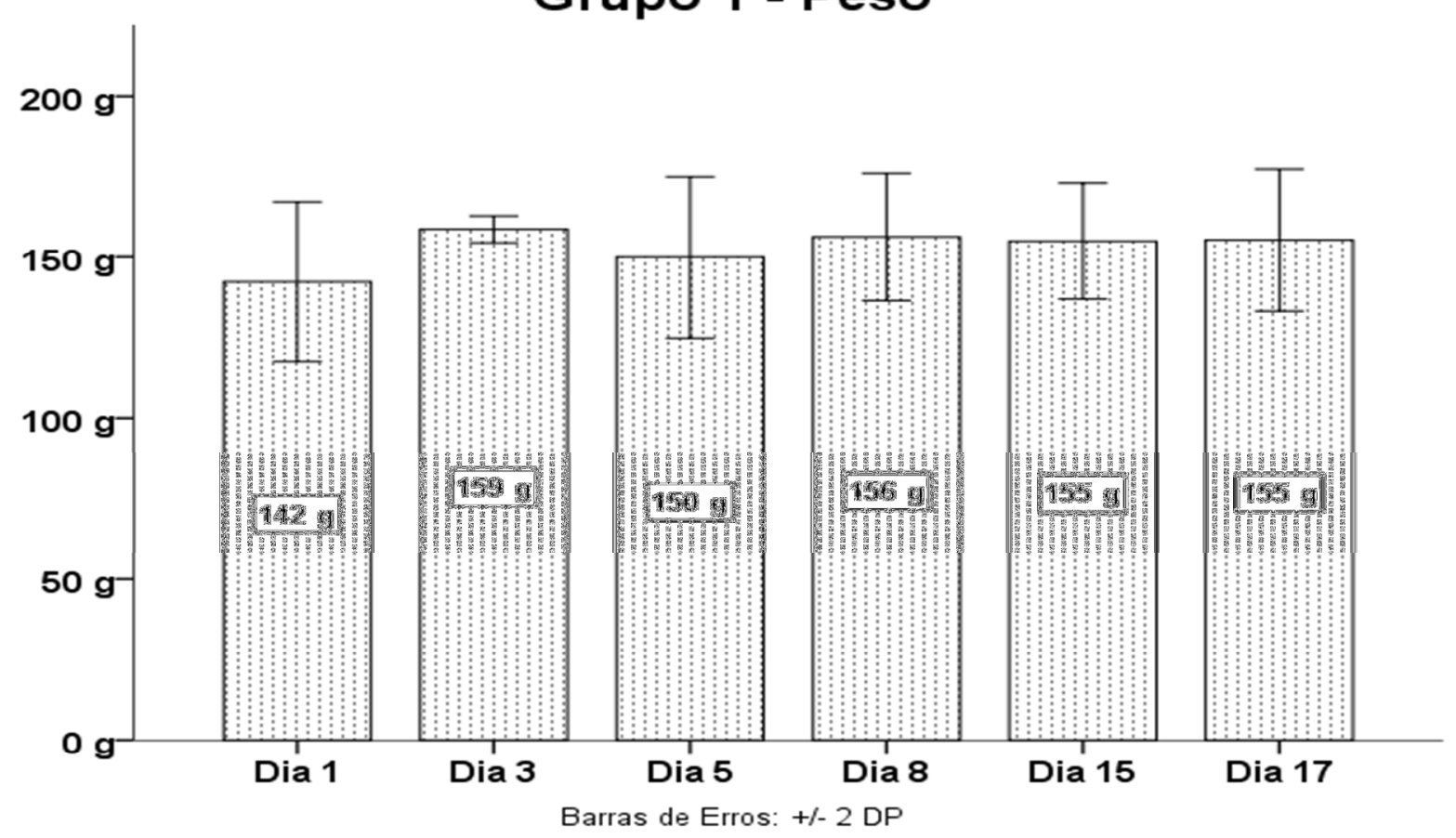

Figura 2A: Análise da massa corporal de ratos diabéticos que utilizam $200 \mathrm{mg}$ de Bauhinia forficata. 


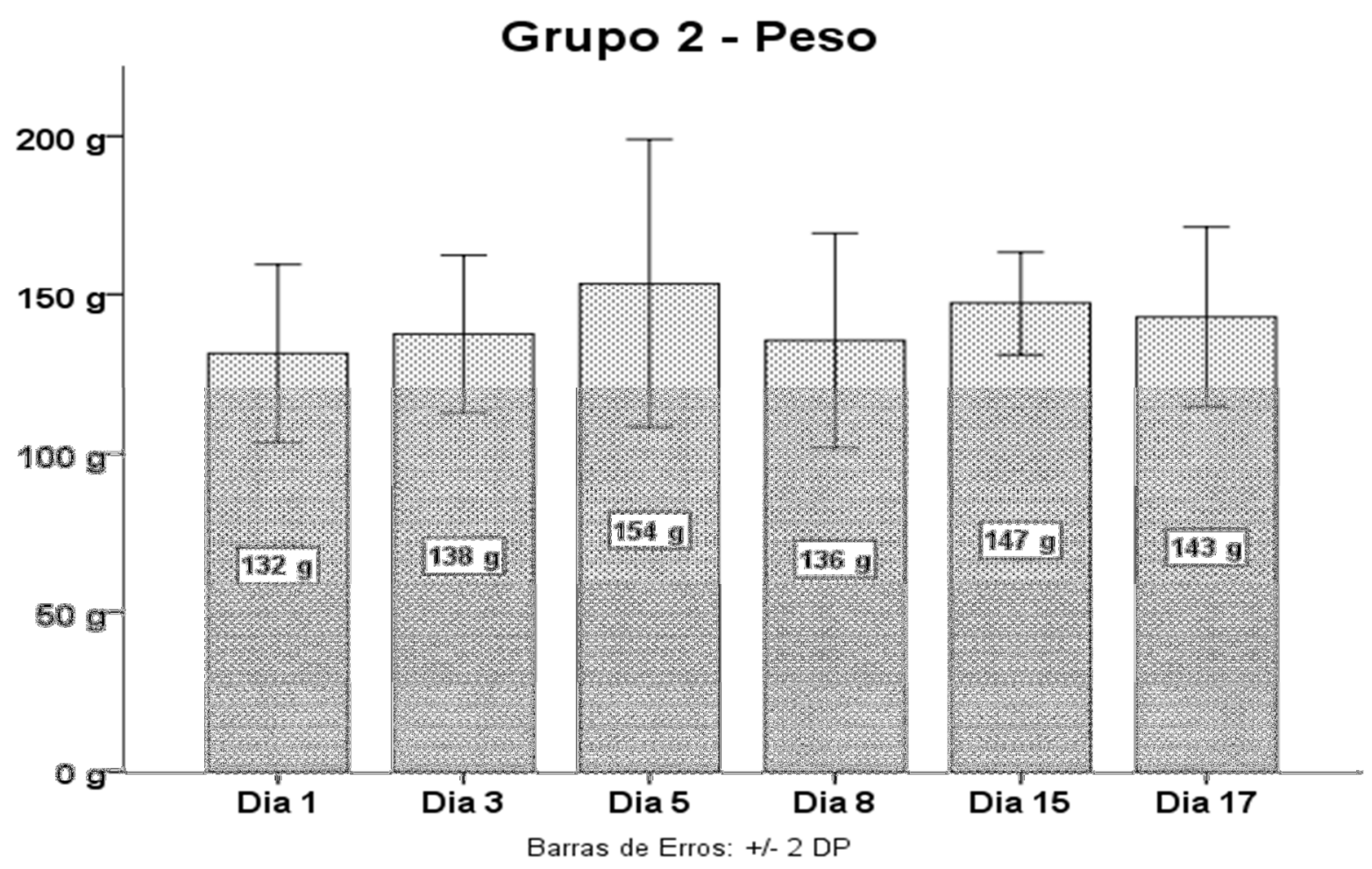

Figura 2B: Análise da massa corporal de ratos diabéticos que utilizam $500 \mathrm{mg}$ de metformina/ $0,2 \mathrm{~mL}$ de insulina. 


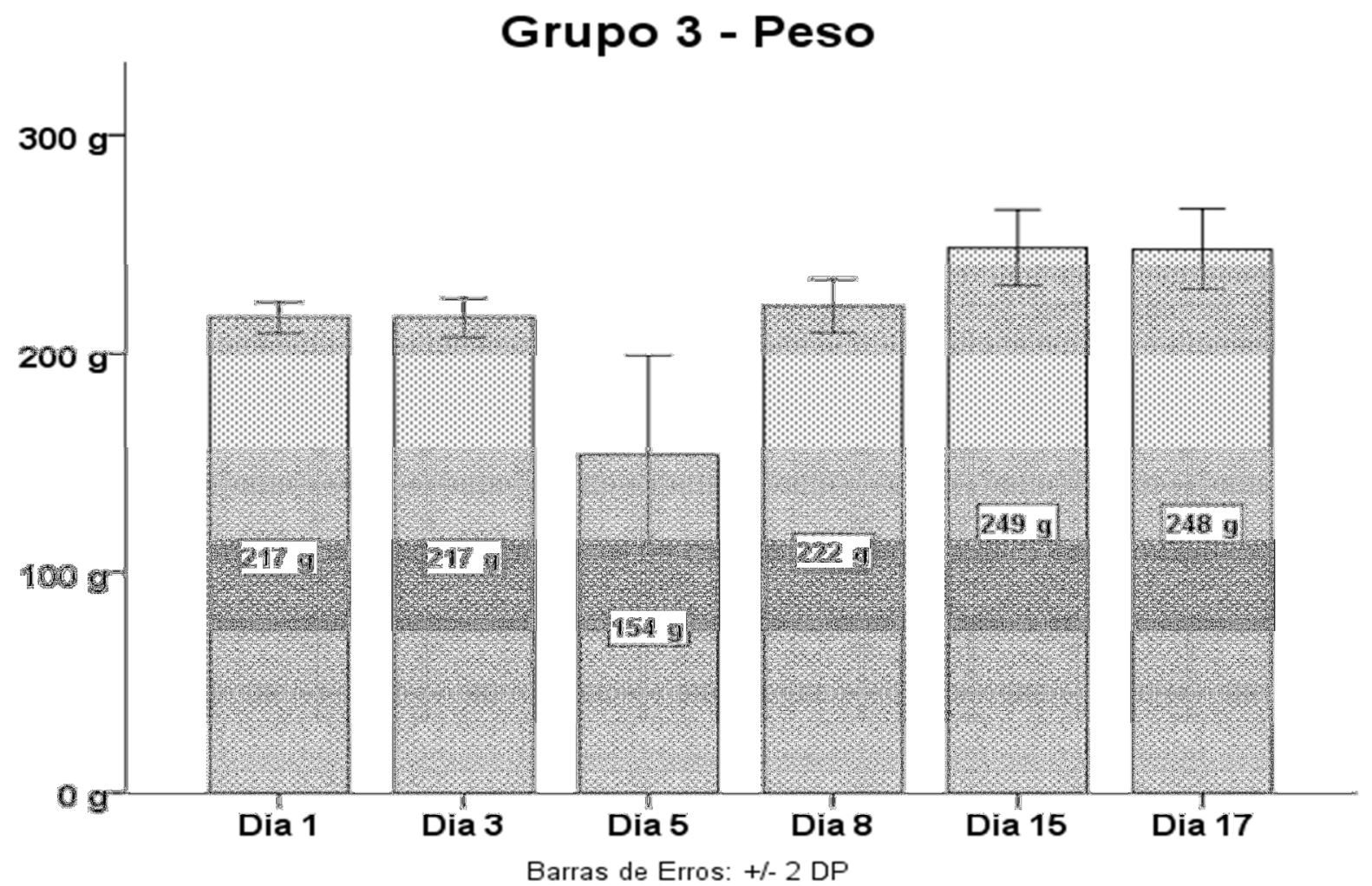

Figura 2C: Análise da massa corporal de ratos diabéticos que utilizam $100 \mathrm{mg}$ de Bauhinia forficata. 


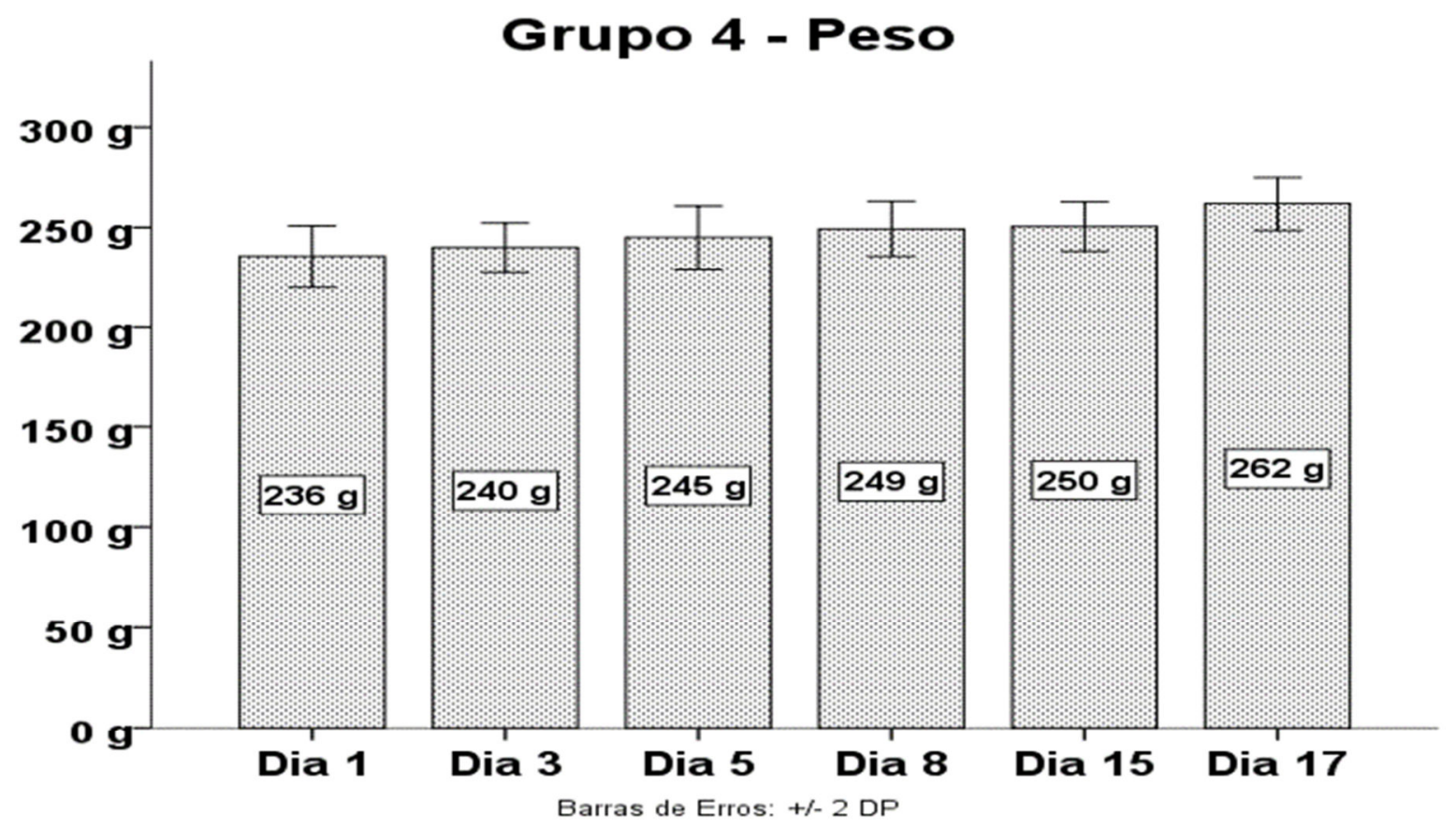

Figura 2D: Análise da massa corporal de ratos não diabéticos para controle.

Na figura 2A, demonstrou a relação do peso em gramas dos ratos do grupo 1, avaliados durante os dias de pesquisa, que foi administrado $200 \mathrm{mg} / \mathrm{kg}$ do extrato, mostrando, que houve aumento de peso de 13 gramas em média pelo grupo, pois iniciou-se com $142 \mathrm{~g}$ e concluiu-se com $155 \mathrm{~g}$ a média de massa corporal.

$\mathrm{Na}$ figura 2B, estão relacionados o peso dos animais em diferentes dias de análise. Este grupo fazia uso da metformina, onde constatou-se a variação da média de peso dos ratos do grupo: tendo início com $132 \mathrm{~g}$ e terminando com $143 \mathrm{~g}$ demonstrando que houve o aumento de $9 \mathrm{~g}$ em sua média do grupo, baixa variância em comparação com os outros grupos.

$\mathrm{Na}$ figura $2 \mathrm{C}$, ilustra-se o peso dos ratos com os dias que foram analisados, $\mathrm{O}$ grupo fazia o uso de $100 \mathrm{mg} / \mathrm{kg}$ do extrato, observando-se que houve aumento significativo na média de peso da massa corporal dos animais, com uma queda significativa no $5^{\circ}$ dia de análise onde revela que o aumento de peso não foi gradativo crescente, ora aumentava, ora diminuía, tendo iniciado com $217 \mathrm{~g}$ e terminado com $248 \mathrm{~g}$, essa variância é bastante significativa com o aumento de $31 \mathrm{~g}$ em média de peso do grupo. 
$\mathrm{Na}$ figura 2D, observa-se o peso dos ratos com os dias de verificação do grupo 4, também chamado de grupo controle, onde não foi utilizado extrato e nem houve a indução ao diabetes, ou seja, modelos saudáveis para comparação. Sua média de peso do grupo mostrou-se bem significativa pelo fato de seus animais manter-se saudáveis em toda a pesquisa, salientando-se que estes animais permaneciam com aumento de peso bem maior que ratos diabéticos, tendo este aumento de forma gradativa e crescente, iniciando-se com a média de $236 \mathrm{~g} \mathrm{e}$ concluindo-se com $262 \mathrm{~g}$, com aumento de $30 \mathrm{~g}$ da média de peso do grupo.

Consta na literatura científica alguns relatos sobre a ação antioxidante da Bauhinia forficata, que está relacionada à presença de flavonoides, que compõem o grupo dos vegetais antioxidantes. Com isso, ela surtiu efeito positivo em algumas patologias, e foi-se comprovado que através da utilização das folhas em forma de chás, pôde-se ter resultados satisfatórios no tratamento da diabetes melittus e suas complicações. O extrato aquoso é o método mais utilizado na utilização de vegetais antioxidantes, encontra-se em diversas culturas a utilização do extrato da Bauhinia forficata. Faz-se necessário novas pesquisas para salientar e comprovar a eficiência desta erva, além de buscar propriedades quanto as doses de sua utilização (MARQUES et al., 2013).

No estudo de Marques et al. (2013) foram apresentados os primeiros relatos da utilização da B. forficata em pacientes diabéticos, entre 1929 e 1931, com resultados amplamente positivos. A partir de então, os extratos da planta têm sido utilizados de forma abrangente em diversos países e até no Brasil. Outras atividades farmacológicas relacionadas a Bauhinia forficata têm sido estudadas e utilizadas na medicina popular, como diurético, tônico, no combate a elefantíase e depurativo. Além desses, constam-se também estudos no que diz respeito a ação hipocolesterolemiante e antilipidêmica. Alguns autores relatam atividades satisfatórias no tratamento de Alzheimer, por ter inibição parcial das colinesterases. 


\section{CONCLUSÃO}

Diante do exposto, verificou-se que os estudos pautados na utilização da Bauhinia forficata na pesquisa do controle terapêutico do diabetes mellitus tem como resultados positivos eficácia na diminuição da glicemia. Dessa forma, a linha de pesquisa deve ser continuada e um maior suporte e atenção devem ser empregados à mesma, tanto para as pesquisas realizadas dentro do tema, mas também ao emprego da aplicação de seus resultados, como para validação da utilização da planta na medicina popular, podendo, assim, gerar uma maior contribuição e de forma direta e ativa com a sociedade que carece cada vez mais de novos fitoterápicos para tratamento e controle desta patologia agressiva.

\section{REFERÊNCIAS BIBLIOGRÁFICAS}

BRINATI, Lídia Miranda et al. Prevalência e fatores associados à neuropatia periférica em indivíduos com diabetes mellitus Prevalence and factors associated with peripheral neuropathy in individuals with diabetes mellitus. Revista de Pesquisa: Cuidado é Fundamental Online, v. 9, n. 2, p. 347-355, 2017.

BUSZNIEZ, Patricia et al. Caracterización fisicoquímica, óptica y reológica de soluciones extractivas de phyllanthus sellowianus y bauhinia forficata. In: Anales (Asociación Física Argentina). Asociación Física Argentina, 2017. p. 66-69.

COSTA, Amine Farias et al. Carga do diabetes mellitus tipo 2 no Brasil. Cadernos de Saúde Pública, v. 33, p. e00197915, 2017.

FIGUEIREDO, F. J.; LIMA, V. L. A. G. Antioxidant activity of anthocyanins from quixabeira (Sideroxylon obtusifolium) fruits. Revista Brasileira de Plantas Medicinais, v. 17, n. 3, p. 473$479,2015$.

FORGIARINI JUNIOR, Luiz Alberto et al. Experimental diabetes mellitus: oxidative stress and changes in lung structure. Jornal Brasileiro de Pneumologia, v. 35, n. 8, p. 788-791, 2009.

JARADAT, Nidal Amin et al. Herbal remedies use by breast cancer patients in the West Bank of Palestine. Journal of ethnopharmacology, v. 178, p. 1-8, 2016.

LERCO, M.M.; SPADELLA, C.T.; MACHADO, J.L.M.; SCHELLINI, S.A.; PADOVANI, C.R. Caracterização de um modelo experimental de Diabetes Mellitus, induzido pela aloxana em ratos. Estudo clínico e laboratorial. Acta Cirurgica Brasileira, São Paulo, v.18, n.2, p.132- 142, 2003. 
LEVEAU, Carlos M. et al. Does geographic context matter in diabetes-related mortality? Spatial and time trends in Argentina, 1990-2012. Cadernos de saude publica, v. 33, n. 1, 2017. VISENTIN, Angelita et al. Self-care of users with type 1 diabetes in a basic health unit. Journal of Nursing UFPE on line, [S.I.], v. 10, n. 3, p. 991-998, jan. 2016. ISSN 1981-8963.

LIMA, M.A.; LIMA, L.M.B.; RITA, D.P.C.; NAVARRO, F.C.; TATSUKAWA, R.S.; PEREIRA, G.A.; REIS, L.C.; ABREU, M.E.A.; BORGES, M.F. Análise quantitativa das células das ilhotas pancreáticas em ratos sob efeito de aloxana. Medicina, Ribeirão Preto, v.34, n.3/4, p.308-314, 2001.

GOIS, M. A. F. et al. Etnobotânica de espécies vegetais medicinais no tratamento de transtornos do sistema gastrointestinal. Rev. Bras. PI. Med., Campinas, v. 18, n. 2, p. 547-557, 2016.

MARQUES, Graziella Silvestre et al. Caracterização fitoquímica e físico-química das folhas de Bauhinia forficata Link coletada em duas regiões brasileiras. Revista de Ciências Farmacêuticas Básica e Aplicada, v. 33, n. 1, p. 57-62, 2012.

MARQUES, Graziella Silvestre et al. Estado da arte de Bauhinia forficataLink (Fabaceae) como alternativa terapêutica para o tratamento do diabetes melittus. Revista de Ciências Farmacêuticas Básica e Aplicada, v. 34, n. 3, p. 313-320, 2013.

MESSIAS, M.C.T.B. et al.Uso popular de plantas medicinais e perfil socioeconômico dos usuários: um estudo em área urbana em Ouro Preto, MG, Brasil. Rev. bras. plantas med. 2015, vol.17, n.1, pp.76-104. ISSN 1516-0572.

MOREIRA, Tiago Ricardo et al. Prevalência e fatores associados à doença arterial periférica em indivíduos com diabetes mellitus. Revista de Enfermagem do Centro-Oeste Mineiro, v. 7, 2017.

NETO, F. R. G; ALMEIDA, G.S.S.A.; JESUS, N.G. and FONSECA, M.R.Estudo Etnobotânico de plantas medicinais utilizadas pela Comunidade do Sisal no município de Catu, Bahia, Brasil. Rev. bras. plantas med 2014, vol.16, n.4, pp.856-865. ISSN 1516-0572.

PEREIRA, J. B. A. et al. O papel terapêutico do Programa Farmácia Viva e das plantas medicinais no centro-sul piauiense. Rev. Bras. PI. Med, v. 17, n. 4, p. 550-561, 2015. Ge Li , Binlin Wu , Meliza Ward G., Angie CN Chong, Sushmita Mukherjee, Shuibing Chen, Mingming Hao J Cell Sci 2016 129: 2865-2875.

SIMÕES, Ranggel Carvalho et al. Estudo fitoquímico de Bauhinia forficata (Fabaceae). Biota Amazônia (Biote Amazonie, Biota Amazonia, Amazonian Biota), v. 5, n. 1, p. 27-31, 2015.

SOARES, Julio Cesar Mendes; DA COSTA, Silvio Teixeira; CECIM, Marcelo. Níveis glicêmicos e de colesterol em ratos com Diabetes Mellitus aloxano induzido, tratados com infusão de Bauhinia candicans ou Syzygium Jambolanum Glucose and cholesterol plasma levels in rats with alloxan-induced Diabetes Mellitus treated with infusion of Bauhinia.

TROJAN-RODRIGUES, $\mathrm{M}$ et al. Candicans or Syzygium Jambolanum. Ciência Rural, v. 30, n. 1, p. 113-118, 2000. Plants used as antidiabetics in popular medicine in Rio Grande do Sul, southen Brazil Journal of Ethnopharmacology, v.139, n. 1, p. 155-163, 2012. 